\title{
Aqueous Synthesis of Mesoporous Zr-based Coordination Polymer for Removal of Organic Dye
}

Chenjia Liang, a,\# Junhao Ren, a,\# Samir El Hankari, a, " Jia Huo ${ }^{a, b,{ }^{*}}$

a State Key Laboratory of Chem/Bio-Sensing and Chemometrics, Provincial Hunan Key Laboratory for Graphene Materials and Devices, College of Chemistry and Chemical Engineering, Hunan University, Changsha, 410082, Hunan, China ; ${ }^{b}$ Shenzhen Research Institute of Hunan University, Shenzhen 518057, Guangdong, China

*To whom correspondence should be addressed: E-mail: jiahuo@hnu.edu.cn; elhankari@hnu.edu.cn

\# These authors contributed equally to this paper. 


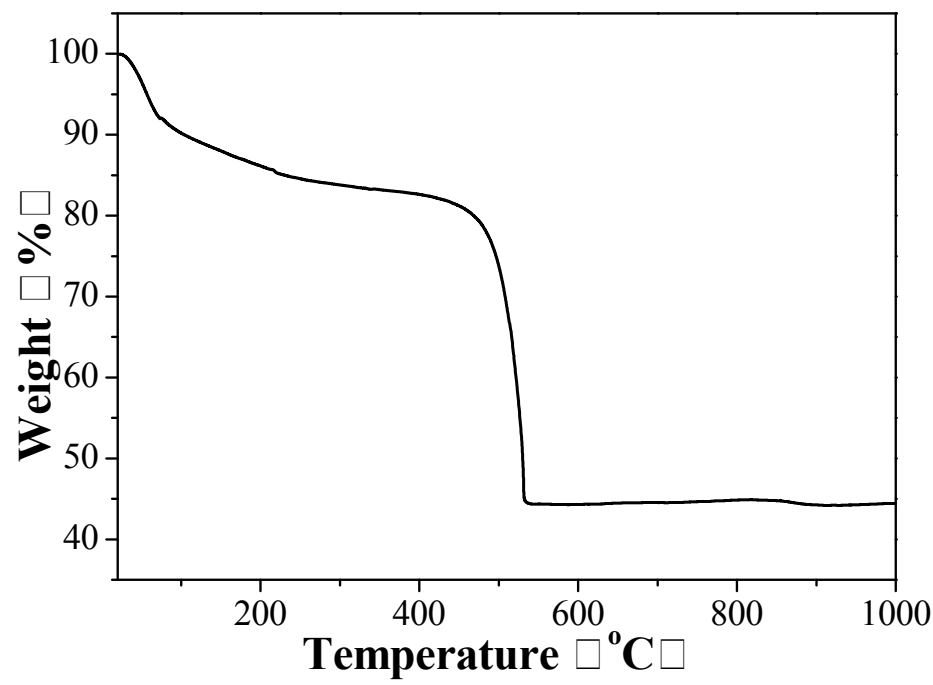

Figure S1. TG curves of $\mathrm{Zr}$-BDC-CP under Ar atmosphere Counts

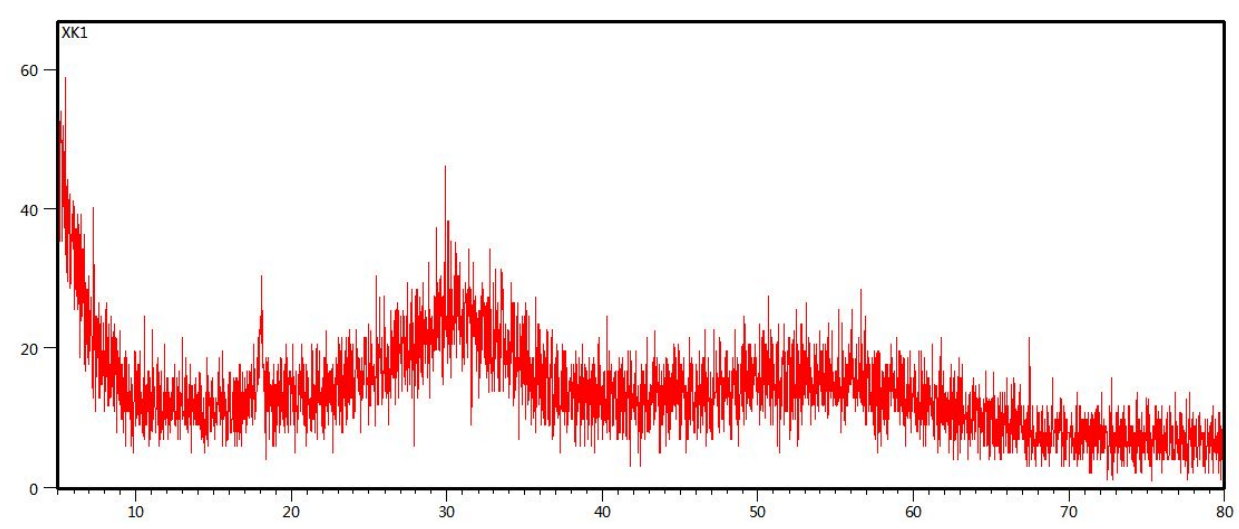

Figure S2. The PRXD pattern of Zr-BDC-CP

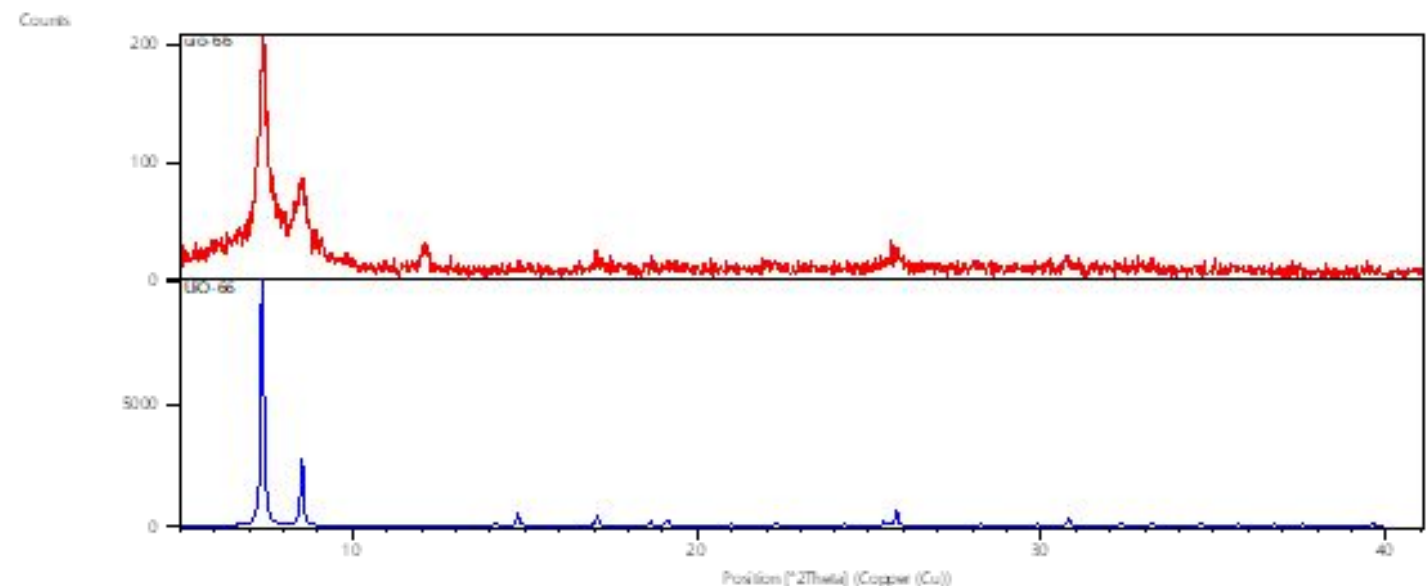

Figure S3. The simulated (bottom) and experimental (upper) PXRD patterns of UIO-66 




Figure S4. The photos of dye solutions after adsorption for Zr-BDC-CP (left) and UIO-66 (right) (the initial dye solution is $100 \mathrm{mg} / \mathrm{L}$ ).

Table S1 Comparison of MB adsorption among different MOFs.

\begin{tabular}{ccccccc}
\hline Material & $\begin{array}{c}\text { Quality } \\
(\mathrm{mg})\end{array}$ & $\begin{array}{c}\text { Concentration } \\
(\mathrm{mg} / \mathrm{L})\end{array}$ & $\begin{array}{c}\text { Volume } \\
(\mathrm{ml})\end{array}$ & $\begin{array}{c}\text { Efficiency } \\
(\%)\end{array}$ & $\begin{array}{c}\text { Amount adsorbed } \\
(\mathrm{mg} / \mathrm{g})\end{array}$ & Ref \\
\hline MOF-235 & 5 & 20 & 50 & 93.5 & 187 & {$[1]$} \\
ZJU-24-0.89 & 5 & 200 & 50 & 45.1 & 902 & {$[2]$} \\
NOTT-101 & 5 & 200 & 50 & 20.0 & 400 & {$[2]$} \\
$\mathrm{Fe}_{3} \mathrm{O}_{4} @$ MIL- & 10 & 75 & 10 & 66.3 & 49.41 & {$[3]$} \\
100(Fe) & 20 & 30 & 20 & - & 11 & {$[4]$} \\
$\begin{array}{c}\text { MIL-101(Cr) } \\
\text { Cu-BTC }\end{array}$ & 5 & 3.2 & 10 & - & 4.4 & {$[5]$} \\
UiO-66 & 50 & 100 & 5 & 35.8 & 3.71 & This \\
Zr-BDC-CP & 50 & 100 & 5 & 94.5 & 9.78 & This \\
Zr-BDC-CP & 50 & 400 & 5 & 93.5 & 38.17 & This \\
& & & & & & work \\
\hline
\end{tabular}

\section{References}

[1] Enamul H.; Jong W.J.; Sung H.J., Adsorptive removal of methyl orange and methylene blue from aqueous solution with a metal-organic framework material, iron terephthalate (MOF-235). J. Hazard Mater. 2011, 185, 507-511.

[2] Zhang Q.; Yu J.C.; Cai J.F.; Song R.J.; Cui Y.J.; Yang Y.; Chen B.L.; Qian G.D., A porous metal-organic framework with - $\mathrm{COOH}$ groups for highly efficient pollutant removal. J. Chem Commun. 2014, 50, 14455-14458.

[3] Shao Y.M.; Zhou L.C.; Bao C.; Ma J.J.; Liu M.Z.; Wang F., Magnetic responsive metalorganic frameworks nanosphere with core-shell structure for highly efficient removal of methylene blue. J. Chem Eng. 2016, 283, 1127-1136.

[4] Shen T.; Luo J.; Zhang S.; Luo X. Hierarchically mesostructured MIL-101 metal-organic 
frameworks with different mineralizing agents for adsorptive removal of methyl orange and methylene blue from aqueous solution. J. Environ. Chem. Eng. 2015, 3, 1372-1383.

[5] Lin S.; Song Z.; Che G.; Ren A.; Li P.; Liu C.; Zhang J. Adsorption behavior of metal-organic frameworks for methylene blue from aqueous solution. Micro. Meso Mater., 2014, 193, 27-34 